\title{
Adaptación y Propiedades Psicométricas del Cuestionario de Violencia Filio- Parental (C-VIFIP) en Jóvenes Chilenos
}

\author{
Adaptation and Psychometric Properties of the Child-to-Parent Violence (CPV-Q) in \\ Young Chileans
}

\author{
Patricia Jiménez-García ${ }^{1}$, Lourdes Contreras $^{2}$, Beatriz Pérez $^{3}$, Félix Cova ${ }^{4}$ y M. Carmen Cano-Lozano ${ }^{5}$
}

\begin{abstract}
Resumen
La violencia filio-parental es un fenómeno de gran transcendencia social debido a su repercusión en las dinámicas familiares. La mayor parte de las investigaciones sobre este tema han sido realizadas en EEUU y España, siendo muy escasas en países de Latinoamérica, como por ejemplo, Chile. El objetivo del presente estudio fue adaptar y analizar las propiedades psicométricas del Cuestionario de Violencia Filio-Parental, versión jóvenes (C-VIFIP) en una muestra de 823 estudiantes universitarios chilenos $\left(M_{\text {edad }}=20,59\right.$; $D T=1,97)$. Los resultados obtenidos apoyan la estructura de cuatro factores de la versión original y muestran adecuadas propiedades psicométricas. Se concluye que este instrumento es útil para la evaluación de la violencia filio-parental en Chile y la alta prevalencia del fenómeno indica la necesidad de continuar la investigación sobre este tipo de violencia en este país.
\end{abstract}

Palabras clave: violencia filio-parental, jóvenes, adaptación de instrumientos, propiedades psicométricas, evaluación

\begin{abstract}
Child-to-parent violence is a phenomenon of great social transcendence due to its repercussion in family dynamics. Most of the research on this topic has been conducted in the USA and Spain, but in Latin American countries it is still scarce, as for example, in Chile. The objective of this study was to adapt and analyse the psychometric properties of the Child-to-Parent Violence Questionnaire, young version (CPV-Q), in a sample of 823 Chilean university students $\left(M_{\mathrm{age}}=20.59 ; S D=1.97\right)$. The results support the four-factor structure of the original version and show adequate psychometric properties. In conclusion, this instrument is useful for the assessment of child-to-parent violence in Chile and the high prevalence of this phenomenon indicates the need to continue researching on this type of violence in this country.
\end{abstract}

Keywords: child-to-parent violence, juveniles, adaptation of instruments, psychometric properties, assessment

\footnotetext{
${ }^{1}$ Estudiante PhD, Departamento de Psicología, Universidad de Jaén, España. Correo: pjg00001@ @red.ujaen.es

${ }^{2} \mathrm{PhD}$, Profesora en Departamento de Psicología, Universidad de Jaén, España.

${ }^{3} \mathrm{PhD}$, Profesora en Núcleo Científico Tecnológico en Ciencias Sociales y Humanidades, Universidad de la Frontera, Temuco, Chile.

${ }^{4} \mathrm{PhD}$, Profesor en Departamento de Psicología, Universidad de Concepción, Chile.

${ }^{5} \mathrm{PhD}$, Profesora en Departamento de Psicología, Universidad de Jaén, España.

Revista Iberoamericana de Diagnóstico y Evaluación - e Avaliação Psicológica. RIDEP · №56 · Vol.3 · 33-46 · 2020

ISSN: 1135-3848 print /2183-6051online
} 


\section{Introducción}

La violencia filio-parental (VFP) se ha convertido en un motivo de preocupación entre profesionales e investigadores, debido al incremento espectacular de este tipo de conductas en los últimos lustros (Contreras \& Cano-Lozano, 2016). Este fenómeno se ha definido como un conjunto de agresiones físicas, psicológicas y económicas del hijo hacia los progenitores, con la intención de conseguir control y poder sobre ellos (Cottrell, 2001), siendo estas agresiones reiteradas en el tiempo (Molla-Esparza \& Aroca-Montolío, 2018).

Respecto a los tipos VFP, Cottrell (2001) describió la violencia física, psicológica y económica. La violencia física se refiere a aquellas conductas como escupir, empujar, abofetear, dar patadas o puñetazos. La violencia psicológica se refiere a aquellas conductas dirigidas a manipular, controlar y herir emocionalmente a los padres (intimidar, escaparse de casa, amenazar, etc.). La violencia verbal es un tipo de maltrato psicológico e incluye acciones como gritar, desafiar, menospreciar, etc. La violencia económica incluye acciones como robar dinero o pertenencias, vender posesiones de los padres, incurrir en deudas que deben pagar los progenitores o exigir a los padres que les compren cosas que no pueden permitirse.

La investigación en torno a la VFP ha experimentado un notable aumento en la última década con objeto de determinar la prevalencia y las variables psicosociales y familiares vinculadas a este tipo de violencia. No obstante, las estimaciones de la prevalencia de este fenómeno son muy dispares, lo que puede deberse a la variabilidad de las muestras objeto de estudio, a la forma de evaluación de VFP, a los tipos de VFP que se incluyen y a los diversos criterios usados para considerar la presencia o ausencia de VFP. Uno de los instrumentos de evaluación más utilizados es la Escala de Tácticas para Conflictos (CTS, Straus, 1979), adaptada en diferentes versiones para evaluar agresiones físicas, psicológicas y verbales hacia los padres (p.e., Beckmann, Bergmann, Fischer, \& Möble, 2017; Gámez-Guadix, Jaureguizar, Almendros, \& Carrobles, 2012). En España, se han desarrollado tres instrumentos específicos para evaluar la VFP.
Uno de ellos es la Escala de Violencia Intrafamiliar, elaborada por Ibabe y Jaureguizar (2011), en el que se evalúa la violencia física, psicológica y emocional. Por su parte, Calvete y Orue (2016) desarrollaron el Cuestionario de Violencia filio-parental Revisado, que evalúa las agresiones físicas y psicológicas e incluye la evaluación de las razones para ejercer VFP.

Más recientemente, se ha elaborado el Cuestionario de Violencia Filio-Parental (CVIFIP), versión adolescentes (Contreras, BustosNavarrete, \& Cano-Lozano, 2019) validado en población española con 1.386 adolescentes $(55,2 \%$ chicas, $44,8 \%$ chicos) con edades comprendidas entre los 12 y los 18 años y la versión jóvenes (Cano-Lozano, Amador-Flores, Valdivia, \& Contreras, 2017) validado con 703 estudiantes universitarios españoles con edades comprendidas entre los 18 y 25 años. En ambos estudios se realizaron análisis de componentes principales seguido de análisis factoriales confirmatorios. En el caso de la versión para adolescentes (Contreras et al., 2019), se extrajo una estructura factorial de cuatro factores correlacionados (violencia psicológica, violencia física, económica y controldominio). En la versión para estudiantes universitarios, el instrumento fue representado por un modelo jerárquico en el que estos cuatro factores de primer orden o dominios específicos (violencia psicológica, violencia física, económica y control-dominio), se encuentran a su vez explicados por un factor de segundo orden, o factor general (Cano-Lozano et al., 2017). A pesar de esta distinción, ambos modelos representan una estructura muy cercana, ya que los modelos construidos en base a la correlación entre sus factores, asumen indirectamente la existencia de un factor superior que explica dichas correlaciones (Herrero, 2010). Adicionalmente, desde una perspectiva teórica, la VFP se configura como un constructo teórico unitario que puede manifestar a través de los diferentes tipos de violencia nombrados (Mackenzie, Podsakoff \& Jarvis, 2005), habiéndose usado en la versión para adolescentes esta conceptualización jerárquica (Contreras, León, \& Cano-Lozano, 2020). En cualquier caso, el factor de control-dominio es la aportación relevante del instrumento original (Contreras et al., 2019), ya que este elemento se encuentra presente en las principales definiciones de VFP y no en los 
instrumentos revisados anteriormente. En los dos instrumentos, los factores de fiabilidad se encontraban entre .74 y .86 y, en la versión adolescentes, se obtuvo una excelente validez externa al relacionarse los factores del C-VIFIP con variables de procesamiento de la información social y la impulsividad (Contreras et al., 2019). Además, el cuestionario también evalúa las razones que motivan este tipo de violencia, identificándose dos tipos de razones: reactivas e instrumentales (Cano-Lozano et al., 2017). Estas se presentan como una subescala independiente con una estructura de dos factores correlacionados (CanoLozano et al., 2017; Contreras et al., 2019). En primer lugar, respecto a la razón de tipo instrumental, que implica el uso de la violencia para obtener lo que el adolescente quiere, la literatura previa señala que los padres víctimas de VFP son percibidos por sus hijos como más permisivos cuando se comparan con padres que no sufren este tipo de violencia (Contreras \& CanoLozano, 2014, 2015), por lo que los adolescentes perciben a sus padres como más débiles y, por tanto, deciden utilizar la violencia para adquirir poder y control sobre las decisiones de la familia (Routt \& Anderson, 2011). En segundo lugar, las razones reactivas se refieren al uso de la violencia en respuesta a una agresión previa o amenaza de agresión. En este sentido, estudios previos indican que la VFP se encuentra relacionada con otras formas de violencia familiar como la violencia de padres hacia hijos (Beckman et al., 2017; Contreras \& Cano-Lozano, 2016; Margolin \& Baucom, 2014) o la exposición a la violencia (Contreras \& CanoLozano, 2016; López-Cepero, Rodríguez-Franco \& Rodríguez-Díaz, 2014).

Respecto a los datos sobre prevalencia de este fenómeno, por un lado, se han realizado estudios con muestras de adolescentes de población general, en los que los participantes informan de su conducta hacia los padres habitualmente durante el último año y, por otro lado, con jóvenes mayores de 18 años a los que se les pide que informen de manera retrospectiva sobre las conductas que realizaban durante la adolescencia. Además, en la mayoría de los estudios se evalúa la presencia de VFP con criterio tolerancia cero (una respuesta diferente a 0 en cualquier ítem, al menos un acto violento en el último año) (García-Díaz et al., 2013). En otros estudios se evalúa la presencia de violencia reiterada, acorde a la reiteración de las conductas violentas que se recoge en las definiciones de la VFP (Cottrell, 2001; MollaEsparza \& Aroca-Montolío, 2018). Concretamente, en las investigaciones realizadas en Estados Unidos y Canadá con muestras de adolescentes de población general (utilizando el criterio de tolerancia cero), se ha encontrado una prevalencia de violencia física que oscila entre el 11 y el $22 \%$ y alrededor del $75 \%$ de violencia verbal (Margolin \& Baucom, 2014). En Alemania se ha encontrado que el $5,5 \%$ de los/las adolescentes ejercen violencia física y el $45 \%$ ejerce violencia verbal hacia sus progenitores (Beckmann et al., 2017). En España, la prevalencia de violencia física oscila entre un 5\%16\% (Calvete \& Orue, 2016; Del Hoyo, GámezGuadix, \& Calvete, 2018; Ibabe, Arnoso, \& Elgorriaga, 2014), mientras que la violencia psicológica oscila entre un 33\%-92\% (Calvete \& Orue, 2016; Del Hoyo et al., 2018; Ibabe et al., 2014). Cuando se evalúa violencia reiterada, los datos descienden drásticamente, encontrándose porcentajes de violencia física en torno al 2\% (Del Hoyo et al., 2018) y entre el 6\%-14\% de violencia psicológica (Calvete \& Orue, 2016; Del Hoyo et al., 2018). Los estudios realizados con jóvenes son mucho más escasos. Utilizando el criterio de tolerancia cero, la prevalencia de violencia física hacia los progenitores oscila entre un 5\%-6\%, mientras que la violencia verbal se estima alrededor de un 72\% (Gámez-Guadix et al., 2012).

Respecto a las diferencias en función del género del agresor, estudios realizados en Estados Unidos y Alemania, señalan que las chicas ejercen más frecuentemente violencia verbal que los chicos hacia sus progenitores (Beckmann et al., 2017; Margolin \& Baucom, 2014). En España, encuentran que las chicas ejercen con mayor frecuencia violencia física y psicológica hacia las madres que los chicos (Calvete et al., 2013; Calvete \& Orue, 2016).

En cuanto al análisis de las diferencias en función del género de la víctima, la literatura indica que son más frecuentes las agresiones físicas (Margolin \& Baucom, 2014) y verbales (Margolin \& Baucom, 2014; Gámez-Guadix et al., 2012) hacia las madres que hacia los padres. En otros estudios, no se encuentran diferencias significativas, pero indican una mayor frecuencia 
en violencia física (Calvete et al., 2013; Calvete \& Orue, 2016) y en violencia psicológica (Calvete et al., 2013; Calvete \& Orue, 2016) hacia las madres en comparación con los padres.

Por otra parte, la investigación sobre este tema en otros países, como por ejemplo en Latinoamérica, es muy escasa y apenas existen estudios sobre este fenómeno. Concretamente, en Chile no existen registros estadísticos oficiales sobre causas judiciales por VFP y, además, son tipificados como violencia de género, violencia doméstica y otros tipos de violencia familiar. Sin embargo, esto no significa que la VFP no esté presente en este país. En dos estudios recientes sobre el tema, se han encontrado porcentajes de violencia física que oscilan entre el $2,4 \%$ y $11,9 \%$ hacia la madre y el $3,8 \%$ y $5,8 \%$ hacia el padre, mientras que los porcentajes de violencia psicológica oscilan entre el $41,7 \%$ y $95,9 \%$ en el caso de la madre y entre el $24 \%$ y $92,5 \%$ en el caso del padre. Respecto a la violencia económica, los porcentajes se sitúan entre el $18,4 \%$ y el $61,5 \%$ hacia madre y entre el $12,4 \%$ y el $63,8 \%$ hacia el padre (Álvarez, Sepúlveda, \& Espinoza, 2016; Ilabaca \& Gaete, 2018). Asimismo, cuando se evalúa la presencia de violencia reiterada, se observa una prevalencia de violencia física de $0,2 \%$ y $6,5 \%$ hacia la madre y hacia el padre, un $14,1 \%$ y $11,4 \%$ de violencia psicológica hacia la madre y hacia el padre y un $10,6 \%$ y $12,6 \%$ de violencia económica hacia la madre y hacia el padre, respectivamente (Ilabaca $\&$ Gaete, 2018).

En consecuencia, debido a la escasez de estudios sobre VFP en Chile, en este trabajo se planteó, en primer lugar, la adaptación del Cuestionario de Violencia Filio-Parental, versión jóvenes (C-VIFIP) de Cano-Lozano et al. (2017) y el análisis de sus propiedades psicométricas (estructura, fiabilidad y validez), en una muestra de jóvenes chilenos. Además, se analizó la prevalencia de la VFP en jóvenes chilenos y las razones para agredir a sus progenitores.

\section{Método}

\section{Participantes}

La muestra se encuentra compuesta por un total de 823 participantes universitarios del sur de Chile. La edad media se sitúa en 20,59 años
( $M=20,59 ; D T=1,97$; Rango 18-25 años), siendo mujeres el 60,8\% ( $n=500)$ de la muestra. Entre los 12 años y los 17 , el 67,6\% ( $n=554)$ convivió con sus padres, el $21 \%(n=172)$ solo con su madre, y el 7,3\% ( $n=60)$ con su madre y la pareja de esta. El resto $(4 \%, n=33)$, con otros familiares. En mismo periodo, la situación económica de la familia era media-suficiente para la mayor parte de la muestra $(68,9 \%, n=566)$. El 19,1\% $(n=157)$ tuvo una situación económica media-alta, mientras que para el sobrante $(11,9 \%, n=98)$ fue baja-insuficiente.

\section{Instrumentos}

Breve cuestionario ad hoc con el fin de obtener los datos socio-demográficos de los participantes. Se realizaron un conjunto de preguntas relativas a la edad, género, estado civil de los progenitores (casados; divorciados/separados; viudo/a; nunca han vivido juntos), convivencia familiar (ambos padres; padre; madre) y nivel socioeconómico. Respecto al nivel socioeconómico, se pidió a los participantes que respondieran de acuerdo a los siguientes niveles: un ingreso bajo-insuficiente, es un nivel en el que la familia tiene dificultades para satisfacer las necesidades de comida, abrigo y vestimenta. Los ingresos medios-suficientes son aquellos que permiten un nivel de vida cómodo y seguridad económica. Los ingresos medios-altos se refieren a situaciones en las que el ingreso familiar permite un alto estándar de vida que incluye algunos lujos y comodidades.

Cuestionario de Violencia Filio-Parental, versión jóvenes (C-VIFIP, Cano-Lozano et al., 2017), que evalúa conductas físicas, psicológicas y económicas, así como conductas de control y dominio sobre los padres, aspecto esencial en la definición de VFP (Cottrell, 2001) y que no está incluido en instrumentos previos. Se compone de 19 ítems referidos al padre y 19 ítems referidos a la madre, en un formato de respuesta tipo Likert: 0 (nunca), 1 (raramente $=$ ha ocurrido alguna vez), 2 (algunas veces $=2-3$ veces), 3 (bastantes veces $=4$ 5 veces) y 4 (muy a menudo=6 veces o más). Cada hijo/a informa de su conducta de forma retrospectiva en relación con la madre y el padre, respecto al periodo comprendido entre los 12 y 17 años. También se incluyen ocho ítems que informan acerca de las razones para llevar a cabo 
las conductas violentas en un formato de respuesta tipo Likert: $O$ (nunca), 1 (algunas veces), 2 (casi siempre) y 3 (siempre).

Inventario de Dimensiones de Disciplina (IDD; Straus \& Fauchier, 2007) que evalúa el contexto y el modo de implementación de la disciplina parental. De este instrumento se utilizó la escala de apoyo y afecto (que consta de 3 ítems) de la sección D y la escala de castigo corporal (consta de 4 ítems), agresión psicológica (consta de 4 ítems) y control-supervisión (consta de 2 ítems) de la sección C. Los ítems se responden haciendo referencia al padre y a la madre, mediante una escala tipo Likert con cinco anclajes.

\section{Procedimiento}

Se obtuvo el informe favorable de la Comisión de Ética de la Universidad de Jaén (España) y la autorización de las autoridades universitarias respectivas de cada una de las diferentes universidades en Chile que participaron en el estudio. Antes de iniciar el estudio, se llevó a cabo una adaptación del lenguaje del instrumento con un experto cualificado para examinar las diferencias lingüísticas, psicológicas y culturales entre las poblaciones de interés, con la finalidad de realizar una corrección lingüística y adecuación práctica para hacer el instrumento más comprensible a los estudiantes chilenos. Asimismo, se hizo una aplicación piloto con 30 estudiantes universitarios de Psicología para detectar posibles errores o dificultades de comprensión de los ítems. El estudio piloto mostró que los ítems se comprendían sin dificultad. La evaluación, a través de un muestreo intencional, se realizó de forma grupal en las aulas universitarias. Los participantes recibieron una hoja de información sobre la investigación y firmaron un documento de consentimiento. La participación fue anónima y voluntaria.

\section{Análisis estadísticos}

Los análisis fueron realizados con los programas estadísticos SPSS 23.0 y Mplus 7. En primer lugar, se procedió a evaluar la estructura interna del instrumento C-VIFIP original (CanoLozano et al., 2017) en la muestra del presente trabajo, estableciendo que las conductas violentas, medidas a través de los ítems del instrumento, son manifestaciones del constructo teórico presente en el individuo (Mackenzie et al.,2005), tratándose, por tanto, de un modelo reflectivo (Henseler, 2017). Para la escala de razones, se consideró un modelo de dos factores correlacionados (razones instrumentales y razones reactivas). Con el objetivo de determinar qué estructura factorial se ajusta mejor para la escala de violencia hacia la madre y el padre, se explora la estructura de cuatro factores correlacionados (Contreras et al., 2019) y la estructura jerárquica con cuatro factores de primer orden o dominios específicos, y un factor de segundo orden o factor general (Cano-Lozano et al., 2017). Además, se consideró incluir el análisis de la estructura interna como modelo bifactor (Rodríguez, Reise \& Haviland, 2016). Una alternativa al modelo jerárquico que permite considerar en un mismo modelo, un factor general como construcción central más amplia que explica la covarianza de todas las variables observadas, al mismo tiempo que se representan subdominios conceptuales más específicos. Además, este modelo permite juzgar si el instrumento sometido a análisis tiene un factor general suficientemente fuerte que justifique la adecuación de una puntuación total (Reise, 2012; Rodríguez et al., 2016)

Como estrategia analítica se utiliza el Análisis Factorial Confirmatorio (AFC), considerando el estimador de Mínimos Cuadrados No Ponderados Robusto (ULSMV) sobre una matriz policórica, debido a la naturaleza ordinal de los datos. Adicionalmente, y debido a la necesidad de ajuste de la estructura propuesta en la literatura a la muestra de estudio mediante la correlación de varianza de dos errores, se utilizó la técnica de validez cruzada en la subescala de razones (Arlot \& Celisse, 2010). Se valoró el ajuste del modelo mediante los índices de ajuste Chi-Cuadrado corregido de Satorra-Bentler, RMSEA, CFI y TLI. Buen ajuste: CFI y TLI $\geq .95$, y RMSEA $<.05$. Ajuste aceptable: CFI y TLI $\geq .90$, y RMSEA $<.08$ (Kline, 2005).

Posteriormente, para estimar la fiabilidad, se utilizaron el coeficiente omega de McDonald y el omega jerárquico, dependiendo de la naturaleza unidimensional o multidimensional de la escala, además de los valores PUC (porcentaje de correlaciones no contaminadas) y ECV (varianza común explicada) (Reise, Scheines, Widaman, \& 
Tabla 1. Estadísticos descriptivos del Cuestionario de Violencia Filio-parental (C-VIFIP)

\begin{tabular}{|c|c|c|c|c|c|c|c|c|c|c|}
\hline \multirow[b]{2}{*}{ Item } & \multirow[b]{2}{*}{ Factor } & \multicolumn{4}{|c|}{ Padre } & & \multicolumn{4}{|c|}{ Madre } \\
\hline & & $M$ & $D T$ & Asimetría & Curtosis & & $M$ & $D T$ & Asimetría & Curtosis \\
\hline 1 & \multirow{6}{*}{ I } & 1,51 & 1,11 & 0,34 & $-0,68$ & & 1,78 & 1,15 & 0,32 & $-0,71$ \\
\hline 3 & & 0,59 & 0,93 & 1,61 & 2,00 & & 0,52 & 0,93 & 1,61 & 2,01 \\
\hline 4 & & 0,84 & 0,99 & 1,06 & 0,45 & & 0,85 & 1,02 & 1,08 & 0,38 \\
\hline 6 & & 0,89 & 1,02 & 1,13 & 0,69 & & 0,85 & 0,99 & 1,16 & 0,82 \\
\hline 7 & & 0,33 & 0,76 & 2,72 & 7,55 & & 0,40 & 0,81 & 2,25 & 4,85 \\
\hline 18 & & 0,98 & 1,18 & 1,04 & 0,12 & & 0,99 & 1,12 & 0,97 & 0,09 \\
\hline 9 & \multirow{6}{*}{ II } & 0,09 & 0,39 & 4,98 & 28,32 & & 0,10 & 0,40 & 4,49 & 22,55 \\
\hline 11 & & 0,06 & 0,34 & 6,80 & 52,75 & & 0,04 & 0,24 & 7,04 & 57,26 \\
\hline 12 & & 0,02 & 0,18 & 11,04 & 140,98 & & 0,01 & 0,10 & 13,65 & 207,39 \\
\hline 14 & & 0,08 & 0,36 & 5,83 & 40,38 & & 0,06 & 0,33 & 7,03 & 58,23 \\
\hline 16 & & 0,15 & 0,51 & 4,22 & 20,03 & & 0,14 & 0,47 & 3,94 & 17,83 \\
\hline 2 & & 0,20 & 0,56 & 2,97 & 8,72 & & 0,23 & 0,57 & 2,72 & 7,47 \\
\hline 5 & \multirow{3}{*}{ III } & 0,35 & 0,75 & 2,41 & 5,85 & & 0,36 & 0,77 & 2,39 & 5,52 \\
\hline 13 & & 0,89 & 0,99 & 0,97 & 0,35 & & 0,99 & 2,03 & 0,88 & 0,08 \\
\hline 15 & & 0,48 & 0,86 & 1,99 & 3,84 & & 0,50 & 0,87 & 1,88 & 3,28 \\
\hline 8 & \multirow{4}{*}{ IV } & 0,38 & 0,79 & 2,45 & 6,06 & & 0,39 & 0,39 & 2,42 & 5,63 \\
\hline 10 & & 0,73 & 1,02 & 1,35 & 1,09 & & 0,84 & 0,84 & 1,17 & 0,46 \\
\hline 17 & & 0,19 & 0,56 & 3,63 & 14,93 & & 0,25 & 0,25 & 2,90 & 9,07 \\
\hline 19 & & 0,44 & 0,79 & 2,06 & 4,44 & & 0,55 & 0,55 & 1,72 & 2,69 \\
\hline Razones & \multicolumn{5}{|c|}{ Subconjunto 1} & & \multicolumn{4}{|c|}{ Subconjunto 2} \\
\hline 1 & \multirow{5}{*}{ RI } & 2,27 & 0,86 & 0,39 & $-0,43$ & \multirow{5}{*}{ RI } & 2,27 & 0,85 & 0,31 & $-0,47$ \\
\hline 2 & & 2,04 & 0,76 & 0,62 & 0,45 & & 2,05 & 0,74 & 0,51 & 0,24 \\
\hline 3 & & 2,12 & 0,77 & 0,48 & 0,11 & & 2,13 & 0,77 & 0,43 & 0,01 \\
\hline 4 & & 2,30 & 0,81 & 0,32 & $-0,29$ & & 2,30 & 0,82 & 0,31 & $-0,34$ \\
\hline 5 & & 2,04 & 0,72 & 0,68 & 0,84 & & 2,04 & 0,73 & 0,66 & 0,74 \\
\hline 6 & \multirow{3}{*}{$\mathrm{RR}$} & 2,88 & 0,95 & $-0,29$ & $-0,95$ & \multirow{3}{*}{$\mathrm{RR}$} & 2,83 & 0,94 & $-0,26$ & $-0,94$ \\
\hline 7 & & 2,09 & 0,82 & 0,81 & 0,45 & & 2,07 & 0,79 & 0,78 & 0,53 \\
\hline 8 & & 2,52 & 0,94 & 0,11 & $-0,90$ & & 2,46 & 0,93 & 0,19 & $-0,85$ \\
\hline
\end{tabular}

Nota. I: violencia psicológica; II: violencia física; III: violencia económica; IV: violencia control-dominio; RI: razones instrumentales; RR: razones reactivas

Havilandm, 2013; Rodríguez et al., 2015) para valorar la pertinencia de la unidimensionalidad en bifactor.

Para analizar la prevalencia de la VFP en los jóvenes chilenos, se evaluaron los porcentajes de los jóvenes que han ejercido conductas constitutivas de VFP y se analizaron las diferencias entre los tipos de VFP hacia el padre y hacia la madre utilizando la prueba Chi Cuadrado de McNemar para dos muestras relacionadas y las diferencias entre los tipos de VFP en función del género del agresor utilizando la prueba Chi Cuadrado de Pearson. Se evaluó la presencia de violencia reiterada, siendo el criterio obtener puntuaciones de dos o más (ha ocurrido algunas veces; $2-3$ veces en el último año) en las escalas correspondientes al C-VIFIP para la presencia de conductas constitutivas de VFP psicológica y física. Finalmente, se utilizó la prueba $t$ de Student para analizar las diferencias de medias en las razones para agredir a sus progenitores en función del género del agresor.

Por último, con la finalidad de buscar evidencia externa de validez (validez convergente) se calcularon las correlaciones entre las puntuaciones de cada dimensión del C-VIFIP con las puntuaciones de las escalas del IDD, ya que estudios previos han encontrado que la VFP psicológica y física se relacionan con el empleo de estrategias punitivas como el castigo físico y psicológico (Gámez-Guadix et al., 2012; Del Hoyo et al., 2018), así como con el control de los límites y normas (Gámez-Guadix et al., 2012) y la baja afectividad (Contreras \& Cano-Lozano, 2016). Respecto a las razones para la VFP, la función de la agresión ha sido tradicionalmente caracterizada en términos de agresión instrumental y reactiva, que están relacionados con la exposición a la violencia y con las estrategias punitivas (Contreras \& CanoLozano, 2016; Contreras et al., 2019). 
Tabla 2. Índices de ajuste para las estructuras propuestas en las subescalas de instrumento C-VIFIP

\begin{tabular}{lccccc}
\hline & $\chi^{2}$ & gl & CFI & RMSEA & TLI \\
\hline Subescala Padre & & & & & \\
4 factores correlacionados & 308,69 & 146 & .938 & $.039(0,033-0,045)$ & 0.928 \\
Factor de Segundo Orden & 308,82 & 148 & .939 & $.038(0,032-0,044)$ & 0.930 \\
$\quad$ Bifactor & 225,99 & 129 & .963 & $.032(0,025-0.039)$ & 0.951 \\
Subescala Madre & & & & & \\
4 factores correlacionados & 204,78 & 146 & .955 & $.022(0,015-0,029)$ & 0.946 \\
Factor de Segundo Orden & 208,79 & 148 & .953 & $.022(0,015-0,029)$ & 0.946 \\
$\quad$ Bifactor (VG y Física*) & 195,76 & 128 & .948 & $.026(0,018-0.032)$ & 0.930 \\
Subescala Razones & 156,19 & 19 & .985 & $.095(0,081-0,109)$ & 0.978 \\
2 factores correlacionados. Subconjunto 1. E8 y E7* & 44,58 & 18 & .994 & $.060(0,038-0,083)$ & 0.991 \\
2 factores correlacionados. Subconjunto 2. E8 y E7* & 46,15 & 18 & .994 & $.063(0,041-0,085)$ & 0.991 \\
\hline${ }^{*}$ Correción: $p<.000$ para tos los modelos & & & & &
\end{tabular}

"Corrección: $p<.000$ para todos los modelos

\section{Resultados}

En la Tabla 1 se muestran los estadísticos descriptivos de las pruebas aplicadas.

\section{Análisis Factorial Confirmatorio}

El AFC arrojó valores aceptables en los índices de ajuste para el modelo de cuatro factores correlacionados y el modelo jerárquico con un factor de segundo orden, para ambas subescala (Tabla 2). Se observa entre ambos un ajuste similar, incluso idéntico para los índices CFI, RMSEA y TLI en el caso de la subescala del padre. El modelo bifactor arroja buen ajuste para la escala del padre y aceptable para la escala de la madre, aunque para evitar problemas de convergencia fue preciso liberar la ortogonalidad del factor general lo que produjo algún caso heywood por sobreparametrización. Puesto que el modelo bifactor es similar al modelo jerárquico en su conceptualización, debido a los problemas de convergencia, se optó por la solución jerárquica (ver Figura 1 y 2).

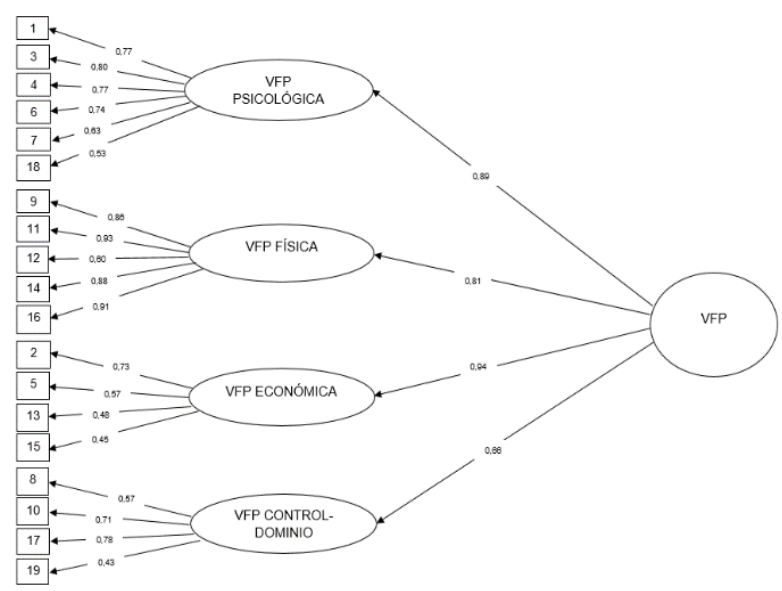

Figura 1. Modelo empírico para la subescala del padre del C-VIFIP. VFP: Violencia filio-parental.

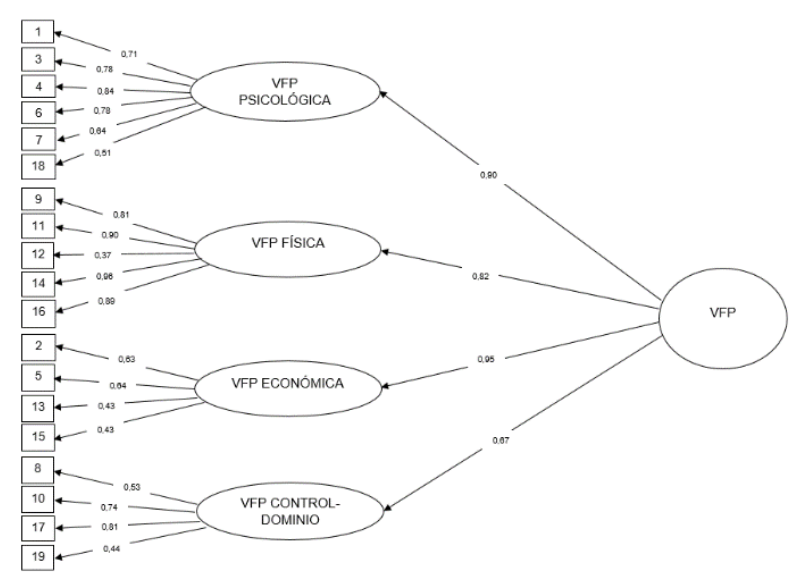

Figura 2. Modelo empírico para la subescala de la madre del C-VIFIP. VFP: Violencia filio-parental

Ambas subescalas presenta un valor de .72 para el coeficiente OmegaH. Los coeficientes OmegaHS para la subescala del padre son: Psicológica .40; Física .78; Económica .48; Control/Dominio .33. Para la subescala de la madre: Psicológica .46; Física .81; Económica .48; Control/Dominio .65. Finalmente, el PUC obtiene un valor aceptable de .78 en ambas subescalas, mientras que el porcentaje de varianza común que es atribuible a un factor general, se acerca al 50\% (ECV subescala padre .47; ECV subescala madre .46), lo que permite asumir un modelo esencialmente unidimensional compatible con la solución jerárquica (Reise et al., 2013).

En lo que respecta a la estructura de dos factores correlacionados propuesta en la literatura para la subescala de razones, se encontró un valor deficiente para el índice de ajuste RMSEA (Ver Tabla 2). Por consiguiente, se procedió a dividir la muestra en dos subconjuntos de manera aleatoria (subconjunto 1, $n=406$; subconjunto 2, $n=400$ ) con el objetivo de analizar la muestra mediante la técnica de validez cruzada. El primer 
Tabla 3. Correlaciones entre las dimensiones del C-VIFIP y las dimensiones del Inventario de Disciplina Parental

\begin{tabular}{|c|c|c|c|c|c|c|c|c|c|c|c|c|}
\hline & & \multicolumn{3}{|c|}{ Padre } & \multicolumn{6}{|c|}{ Madre } & & \\
\hline & & I & II & III & IV & & I & II & III & IV & & \\
\hline I & & 1 & - & - & - & & 1 & - & - & - & & \\
\hline II & & $.73^{* *}$ & 1 & - & - & & $.75^{* *}$ & 1 & - & - & & \\
\hline III & & $.82^{* *}$ & $.75^{* *}$ & 1 & - & & $.85^{* *}$ & $.74^{* * *}$ & 1 & - & & \\
\hline \multirow[t]{2}{*}{ IV } & & $.57^{* *}$ & $.52^{* *}$ & $.69^{* *}$ & 1 & & $.56^{* *}$ & $.55^{* *}$ & $.74^{* *}$ & 1 & & \\
\hline & $\alpha$ & & & & & $\alpha$ & & & & & RI & $\mathrm{RR}$ \\
\hline $\mathrm{CP}$ & .75 & $.23^{* *}$ & $.17^{* * *}$ & $.17^{* * *}$ & $.10^{* * *}$ & .76 & $.25^{* *}$ & $.22^{* *}$ & $.21^{* *}$ & $.09^{*}$ & $.15^{* * *}$ & $.35^{* * *}$ \\
\hline APS & .75 & $.39^{* *}$ & $.19^{* * *}$ & $.24^{* *}$ & $.21^{* * *}$ & .73 & $.35^{* *}$ & $.19^{* *}$ & $.24^{* *}$ & $.22^{* * *}$ & $.16^{* *}$ & $.35^{* * *}$ \\
\hline CSP & .73 & $.17^{* *}$ & $.13^{* *}$ & $.14^{* *}$ & $.11^{* * *}$ & .71 & $.17^{* *}$ & $.09^{*}$ & $.14^{* *}$ & $.09^{* * *}$ & $.14^{* *}$ & $.17^{* * *}$ \\
\hline $\mathrm{AF}$ & .83 & $-.19^{* * *}$ & $-.13^{* *}$ & $-.11^{* *}$ & $-.08^{*}$ & .78 & $-.23^{* *}$ & $-.16^{* *}$ & $-.17^{* *}$ & $-.08^{*}$ & $-.10^{* * *}$ & $-.27^{* *}$ \\
\hline
\end{tabular}

Nota. I: VFP psicológica; II: VFP física; III: VFP económica; IV: VFP control y dominio; CP: Castigo Corporal; APS: Agresión Psicológica; CSP: Control y Supervisión; AF: Apoyo y Afecto.

subconjunto fue sometido a AFC. Mostró un buen ajuste tras la correlación de la varianza de los errores de los ítem 7 y 8 . Esta estructura mostró un buen ajuste en el subconjunto 2 (Ver Tabla 2). El coeficiente omega de McDonald indica una consistencia excelente para el factor de razones instrumentales $(.92$ en ambos subconjuntos) y adecuada para el factor de razones reactivas (.77 en subconjunto uno y .78 en subconjunto 2) (ver Figura 3).

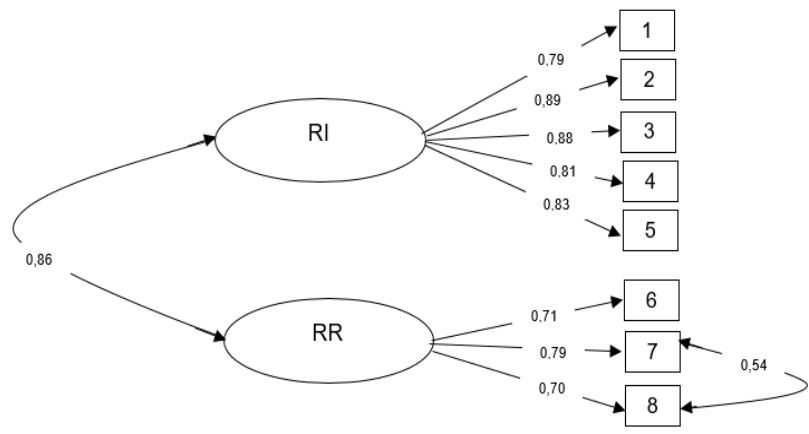

Figura 3. Modelo empírico para la subescala de las razones del C-VIFIP. RI: Razones Instrumentales; RR: Razones Reactivas

Finalmente, se observa en Tabla 3 las correlaciones entre dimensiones para las subescalas de padre y madre. En el caso de la subescala de razones, en ambos subconjuntos se encuentra una correlación estadísticamente significativa $(p<.001)$ entre los factores instrumental y reactivo, con un valor de .87 .

\section{Validez convergente}

Las correlaciones entre las dimensiones del CVIFIP y las dimensiones de la disciplina parental del IDD fueron todas estadísticamente significativas y en la dirección esperada (ver Tabla 3).

\section{Prevalencia de VFP y análisis de las razones de los jóvenes que agreden a sus progenitores}

Los resultados muestran una prevalencia de VFP hacia ambos padres del $76,4 \%$ de violencia psicológica, del 7,4\% de violencia física, del $41 \%$ de violencia económica y del 40,8\% de control y dominio. Respecto a las diferencias según el género de la víctima, se encuentra que la violencia psicológica, económica y el control y dominio se realizan en mayor medida hacia la madre que hacia el padre en lo que respecta a las diferencias según el género. Se observa un mayor porcentaje de violencia psicológica, física, económica y de control y dominio de las chicas hacia las madres respecto a los chicos (ver Tabla 4).

Respecto al análisis de las razones, tal como se observa en la Tabla 5, existen diferencias en cuanto al género del agresor. Las chicas ofrecen más razones de tipo reactivo para la agresión a sus progenitores que los chicos, siendo la razón "por su propio carácter" la más informada por las chicas. 
Tabla 4. Diferencias de medias y porcentajes en los tipos de VFP en función del género del agresor y la víctima

\begin{tabular}{lccccccccccccc}
\hline & \multicolumn{3}{c}{ Padre } & \multicolumn{1}{c}{ Madre } & \multicolumn{4}{c}{ Ambos padres } \\
\hline & $\begin{array}{l}\text { Chicos } \\
\%\end{array}$ & $\begin{array}{c}\text { Chicas } \\
\%\end{array}$ & $\chi^{2}$ & $\mathrm{~V}$ & $\begin{array}{c}\text { Chicos } \\
\%\end{array}$ & $\begin{array}{c}\text { Chicas } \\
\%\end{array}$ & $\chi^{2}$ & \multirow{2}{*}{ V } & $\begin{array}{c}\text { Padres } \\
\%\end{array}$ & $\begin{array}{c}\text { Madres } \\
\%\end{array}$ & $\chi^{2}$ & V \\
\hline VFP Psicológica & 59,6 & 62,0 & 0,48 & .02 & 59,3 & 70,6 & $11,14^{* *}$ & .12 & 61,0 & 66,3 & $8,44^{* *}$ & .45 \\
VFP Física & 4,3 & 5,2 & 0,32 & .02 & 2,5 & 5,6 & $4,54^{*}$ & .07 & 4,9 & 4,5 & 0,90 & .39 \\
VFP Económica & 34,2 & 30,5 & 1,19 & .04 & 33,2 & 37,2 & 1,35 & .04 & 32,0 & 35,7 & $7,25^{*}$ & .68 \\
VFP Control/dominio & 30,7 & 29,9 & 0,06 & .01 & 35,7 & 38,0 & 0,44 & .02 & 30,2 & 37,0 & $26,08^{* *}$ & .69 \\
\hline
\end{tabular}

Nota. VFP: Violencia filio-parental; ${ }^{* *} p<.01 ;{ }^{*} p<.05$

Tabla 5. Razones para ejercer VFP y diferencias entre hijos e hijas

\begin{tabular}{lccc}
\hline & Chicos & \multicolumn{2}{c}{ Chicas } \\
\cline { 2 - 4 } & $M(D T)$ & $M(D T)$ & $t$ \\
\hline Razones Instrumentales & $2,12(0,67)$ & $2,17(0,63)$ & $-1,21$ \\
1 & $2,00(0,85)$ & $2,31(0,85)$ & $-1,73$ \\
2 & $2,01(0,78)$ & $2,06(0,73)$ & $-0,67$ \\
3 & $2,07(0,79)$ & $2,15(0,76)$ & $-1,53$ \\
4 & $2,23(0,83)$ & $2,34(0,81)$ & $-1,87$ \\
5 & $2,06(0,79)$ & $2,01(0,68)$ & 1,06 \\
Razones Reactivas & $2,34(0,73)$ & $2,51(0,73)$ & $-3,28^{* *}$ \\
6 & $2,73(0,98)$ & $2,89(0,91)$ & $-2,27^{*}$ \\
7 & $2,00(0,79)$ & $2,11(0,79)$ & $-1,84$ \\
8 & $2,29(0,89)$ & $2,55(0,95)$ & $-3,89^{* *}$ \\
\hline
\end{tabular}

Nota. VFP: Violencia filio-parental; ${ }^{* *} p<.01 ;{ }^{*} p<.05$

\section{Discusión}

El principal objetivo de este estudio fue adaptar y analizar las propiedades psicométricas del Cuestionario de Violencia Filio-Parental (CVIFIP), versión jóvenes, (Cano-Lozano et al., 2017) en una muestra de jóvenes chilenos. También se planteó analizar la prevalencia de la VFP en jóvenes chilenos y conocer sus razones para agredir a sus progenitores.

Todas las estructuras sometidas a AFC arrojaron un aceptable ajuste. En consonancia con Herrero (2010), el ajuste entre el modelo de cuatro factores correlacionados y el modelo con un factor de segundo orden o jerárquico es muy cercano, ya que los modelos correlacionados asumen indirectamente la existencia de un factor superior. Por otro lado, el modelo bifactor es similar a un modelo jerárquico pero con menos restricciones, lo que da mayor valor a este último cuando su ajuste es superior. Los resultados de este estudio indican que en la práctica, cualquiera de estos modelos puede aplicarse (Reise, 2012). Priorizamos el modelo jerárquico por ser el más adecuado para representar el modelo teórico de la VFP, y coincidente con la estructura propuesta para la versión original del C-VIFIP en población universitaria española (Cano-Lozano et al., 2017).

Este modelo considera un factor general, además de cuatro específicos: violencia psicológica (compuesto por 6 ítems), violencia física (compuesto por 5 ítems), violencia económica (compuesto por 4 ítems) y violencia control y dominio (compuesto por 4 ítems). El factor de violencia control-dominio es una aportación de la versión original del cuestionario C-VIFIP, no presente en otros instrumentos de evaluación de VFP (ver Apéndice).

Por otra parte, el factor general de ambas subescalas muestra una aceptable confiabilidad, mientras que los OmegaHS arrojan valores bajos para las dimensiones, excepto para la violencia física. Este resultado es esperable, ya que este índice basado en la estructura bifactorial, representa las covarianzas entre los ítems después de eliminar la varianza representada por el coeficiente OmegaH (Rodríguez et al., 2016). Además, los valores de OmegaH junto con los valores de PUC y ECV, sugiere que el instrumento presenta una estructura multidimensional compatible con la unidimensionalidad, aunque el valor de ECV no 
alcanza la recomendación mínima de .50 . En definitiva, estos resultados apuntan a que el factor general es suficientemente fuerte para justificar una puntuación total, pero asumiendo un sesgo relativo no tan pequeño, pues la varianza común atribuible a un factor general es del $47 \%$ para la subescala del padre, y del $46 \%$ para la de la madre (Rodríguez et al., 2015, 2016).

Se observa una excelente congruencia entre los factores hacia los ítems referidos al padre como a los referidos a la madre. Asimismo, una contribución del presente estudio es proporcionar información sobre la validez externa, la cual indicó que las conductas de VFP se relacionaron con las estrategias punitivas (Gámez-Guadix et al., 2012; Del Hoyo et al., 2018) y con el control-supervisión de normas y límites (Gámez-Guadix et al., 2012) y el bajo afecto (Contreras \& Cano-Lozano, 2016; Contreras et al., 2019).

Para evaluar la prevalencia de la VFP se establece la presencia de reiteración de conductas violentas, acorde con las principales definiciones sobre este fenómeno (Cottrell, 2001; MollaEsparza \& Aroca-Montolío, 2018). Los resultados del presente estudio indican una prevalencia en VFP psicológica hacia la madre del $59,3 \%$ en chicos y del $70,6 \%$ en chicas y hacia el padre del $59,6 \%$ en chicos y del $62 \%$ en chicas. Respecto a la VFP física, se hallan tasas hacia la madre del $2,5 \%$ en chicos y del $5,6 \%$ en chicas, mientras que hacia el padre se observan tasas del $4,3 \%$ en chicos y del 5,2\% en chicas. Estos resultados difieren a los encontrados en estudios previos que evalúan la presencia reiterada de violencia-respecto a la VFP psicológica en los que encuentran tasas más bajas (Calvete et al., 2013; Calvete \& Orue, 2016), pudiendo ser debido al mayor número de conductas incluidos en este tipo de violencia en el C-VIFIP. En este sentido, cabe indicar que en esta versión del cuestionario se encuentra incluido el ítem "grité a mis padres", siendo una conducta de alta frecuencia durante la adolescencia. Por otra parte, también se observan diferencias en tasas de prevalencia respecto al estudio elaborado por Ilabaca y Gaete (2018) en Chile. En dicho estudio señalan, utilizando la presencia de conductas violentas reiteradas, una prevalencia en violencia física hacia la madre del $0,1 \%$ en chicos y $0,1 \%$ en chicas y hacia el padre del $7,7 \%$ en chicos y $5,4 \%$ en chicas, existiendo una gran diferencia entre las tasas de agresiones hacia el padre y hacia la madre.

Las diferencias entre ambos estudios pueden ser debidas al tipo de criterio restrictivo utilizado y al tipo de conductas evaluadas en los instrumentos de evaluación.

Respecto a las diferencias en el género del agresor, se halla un mayor porcentaje de violencia psicológica y física de las chicas hacia las madres que de los chicos. Este resultado está en línea con estudios previos que revelan un mayor porcentaje de chicas como agresoras respecto a los chicos que ejercen VFP psicológica, verbal y física hacia la madre (Beckmann et al., 2017; Calvete et al., 2013; Calvete \& Orue, 2016; Margolin \& Baucom, 2014).

En cuanto al género de la víctima, en el presente estudio se encuentra que la violencia psicológica se realizan en mayor medida hacia la madre que hacia el padre. Este resultado es consistente con la literatura previa que señalan a la madre como la víctima más frecuente de VFP (Álvarez et al., 2016; Calvete et al., 2013; Calvete \& Orue, 2016; GámezGuadix et al., 2012; Margolin \& Baucom, 2014).

Además, el Cuestionario C-VIFIP permite conocer las razones que llevan a los jóvenes a cometer agresiones contra sus progenitores. Concretamente, se incluyen 8 razones, que se agruparon en dos factores: instrumental y reactivo. Estos dos tipos de razones son halladas también en el estudio de Calvete y Orue (2016) y Contreras et al. (2019). En el presente estudio se obtuvo que las razones de tipo reactivo fueron las más informadas por los jóvenes para agredir a sus progenitores, siendo este resultado consistente con el estudio realizado por Calvete y Orue (2016). En cuanto a las diferencias en el género del agresor, los resultados indican que las razones de tipo instrumental y reactivo son más manifestadas por las chicas que por los chicos, siguiendo en línea con los resultados de Calvete y Orue (2016).

No obstante, este estudio presenta algunas limitaciones que deben tenerse en cuenta a la hora de interpretar los resultados. En primer lugar, en este estudio se utilizaron autoinformes retrospectivos, ya que en dicho cuestionario se les pide a los estudiantes universitarios que respondan en función de la etapa de 12 a 17 años de edad. Sin embargo, Hardt y Rutter (2004) realizaron una revisión empírica sobre información retrospectiva 
dada por participantes acerca de diferentes acontecimientos de la infancia, y encontraron que la información proporcionada sobre hechos ocurridos años atrás es válida. En segundo lugar, sería recomendable realizar un AFC multigrupo para determinar si la configuración del instrumento es invariante para los chicos y las chicas. Finalmente, el modelo bifactor presentó problemas de convergencia, por tanto, se considera necesario realizar nuevos estudios con el objeto de aclarar si la estructura más adecuada para representar el constructo es jerárquica $\mathrm{o}$ bifactor. A pesar de estas limitaciones, el presente estudio proporciona resultados relevantes sobre la evaluación de la VFP en Chile. En este sentido, los resultados indican que el C-VIFIP, versión jóvenes, es un instrumento válido para evaluar breve y fácilmente una amplia variedad de conductas de VFP en sus diferentes formas, incluidas las razones de las agresiones, lo que implica una evaluación integral de la VFP. Este aspecto es esencial para el avance de la investigación, a fin de dar consistencia a los resultados de los estudios sobre este tipo de violencia familiar.

\section{Referencias}

Álvarez, A., Sepúlveda, R., \& Espinoza, S. (2016). Prevalencia de la violencia filioparental en adolescentes de la ciudad de Osorno. Pensamiento y Acción Interdisciplinaria, 1, 59-74.

Arlott, S., \& Celisse, A. (2010). A survey of cross-validation procedures for model selection. Statistics Surveys, 4, 40-79. doi:10.1214/09-SS054.

Beckmann, L., Bergmann, M. C., Fischer, F., \& Möble, T. (2017). Risk and protective factors of child-to-parent violence: A comparison between physical and verbal aggression. Journal of Interpersonal Violence, 1-26. doi:10.1177/0886260517746129.

Calvete, E., Gámez-Guadix, M., Orue, I., González-Diez, Z., López de Arroyabe, E., Sampedro, R., Pereira, R., Zubizarreta, A., \& Borrajo, E. (2013). Brief report: The Adolescent Child-To-Parent Aggression Questionnaire: An examination of aggression against parents in Spanish adolescents. Journal of Adolescence, 36, 1077-1081. doi:10.1016/j.adolescence.

Calvete, E., \& Orue, I. (2016). Violencia filioparental: Frecuencia y razones para las agresiones contra padres y madres. Behavioral Psychology, 24, 481-495.

Cano-Lozano, M. C., Amador-Flores, F., Valdivia, R., \& Contreras, L. (2017). Análisis confirmatorios del cuestionario de violencia filio-parental (C-VIFIP) en jóvenes universitarios. I Congreso Internacional de Psicología, Salud y Educación. Oviedo, España.

Contreras, L., Bustos-Navarrete, C., \& CanoLozano, M. C. (2019). Child-to-parent Violence Questionnaire (CPV-Q): Validation among Spanish adolescents. International Journal of Clinical and Health Psychology, 19, 67-74. doi:10.1016/j.ijchp.2018.09.001.

Contreras, L., \& Cano-Lozano, M. C. (2016). Child-to-parent violence: The role of exposure to violence and its relationship to socialcognitive processing. The European Journal of Psychology Applied to Legal Context, 2, 43-50. doi:10.1016/j.ejpal.2016.03.003.

Contreras, L., León, S., \& Cano-Lozano, M. C. (2020). Socio-cognitive variables involved in the relationship between violence exposure at home and child-to-parent violence. Journal of Adolescence, 80, 19-28. doi:10.1016/j.adolescence.2020.01.017.

Cottrell, B. (2001). Parent abuse: The abuse of parent by their teenage children. Ottawa, Canada: Health Canada, Family Violence Prevention Unit.

Cronbach, L. J. (1951). Coefficient alpha and the internal structure of tests. Psychometrika, 16, 297-334.

Del Hoyo-Bilbao, J., Gámez-Guadix, M., \& Calvete, E. (2018). Corporal punishment by parents and child-to-parent aggression in Spanish adolescents. Anales de Psicología, 34, 108-116.

Gámez-Guadix, M., Jaureguizar, J., Almendros, C., \& Carrobles, J. A. (2012). Estilos de socialización familiar y violencia de hijos a padres en población española. Behavioral Psychology, 20, 585-602. 
García-Díaz, V., Fernández-Feito, A., RodríguezDíaz, F. J., López-González, M. L., MosterioDíaz, M. P., \& Lana-Pérez, A. (2013). Violencia de género en estudiantes de enfermería durante sus relaciones de noviazgo. Atención Primaria, 45, 290-296. doi:10.1016/j.aprim.2012.11.013.

Hardt, J., \& Rutter, M. (2004). Validity of adult retrospective reports of adverse childhood experiences: review of the evidence. Journal of Psychology and Psychiatry, 45, 260-273. doi:10.1111/j.1469-7610.2004.00218.x.

Henseler, J. (2017). Bridging design and behavioral research with variance-based structural equation modeling. Journal of Advertising, $\quad 46, \quad 178-192$. doi:10.1080/00913367.2017.1281780.

Herdoiza-Arroyo, P., \& Chóliz, M. (2018). Impulsividad en la adolescencia: Utilización de una versión breve del cuestionario UPPS en una muestra de jóvenes latinoamericanos y españoles. Revista Iberoamericana de Diagnóstico y Evaluación - e Avaliação Psicológica, $\quad 50, \quad 1-13$. doi:10.21865/RIDEP50.1.10.

Herrero, J. (2010). Confirmatory Factor Analysis in the study of the Structure and Stability of Assessment Instruments: An example with the Self-Esteem Questionnaire (CA-14). Intervención Psicosocial, 19(3), 289-300. doi: 10.5093/in2010v19n3a9

Ibabe, I., Arnoso, A., \& Elgorriaga, E. (2014). Behavioral problems and depressive symptomatology as predictors of child-toparent violence. The European Journal of Psychology Applied to Legal Context, 6, 5361. doi:10.1016/j.ejpal.2014.06.004.

Ilabaca, P., \& Gaete, J. M. (2018). Adolescents who are violent toward their parents: An approach to the situation in Chile. Journal of Interpersonal Violence, 1-21. doi:10.1177/0886260518808856.

Ibabe, I., \& Jaureguizar, J. (2011). ¿Hasta qué punto la violencia filio-parental es bidireccional? Anales de Psicología, 27, 265277.

Izquierdo, I., Olea, J., \& Abad, F. J. (2014). Exploratory factor analysis in validation studies: uses and recommendations. Psicothema, 26, 395-400. doi:10.7334/psicothema2013.349.

Kline, R. B. (2005). Principles and practice of structural equation modeling. New York: Guilford Press.

Lloret-Segura, S., Ferreres-Traver, A., HernándezBaeza, A., \& Tomás-Marco, I. (2014). El análisis factorial exploratorio de los ítems: Una guía práctica, revisada y actualizada. Anales de psicología, 30, 1151-1169. doi:10.6018/analesps.30.3.199361.

López-Cepero, J., Rodríguez-Franco, L., \& Rodríguez-Díaz, F. J. (2014). Evaluación de la violencia de pareja. Una revisión de instrumentos de evaluación conductual. Revista Iberoamericana de Diagnóstico y Evaluación - e Avaliação Psicológica, 40, 37 50.

Mackenzie, S. B., Podsakoff, P. M., \& Jarvis, C. B. (2005). The problem of measurement model misspecification in behavioural and organizational research and some recommended solutions. Journal of Applied Psychology, 90, 710-730. doi:10.1037/0021-9010.90.4.710.

Margolin, G., \& Baucom, B. R. (2014). Adolescents' aggression to parents: Longitudinal links with parents' physical aggression. Journal of Adolescent Health, 55, 645-651. doi:10.1016/j.jadohealth.2014.05.008.

Millsap, R. E., \& Yun-Tein, J. (2004). Assessing Factorial Invariance in Ordered-Categorical Measures. Multivariate Behavioral Research, 39, 479-515. doi:10.1207/S15327906MBR39034.

Molla-Esparza, C., \& Aroca-Montolío, C. (2018). Menores que maltratan a sus progenitores: Definición integral y su ciclo de violencia. Anuario de Psicología Jurídica, 28, 15-21. doi:10.1016/j.apj.2017.01.001.

Reise, S. P. (2012). The rediscovery of bifactor measurement models. Multivariate Behavioral Research, 47(5), 667-696. doi:10.1080/00273171.2012.715555.

Reise, S. P., Scheines, R., Widaman, K. F., \& Haviland, M. G. (2013). Multidimensionality and structural coefficient bias in structural equation modeling a bifactor perspective. Educational and Psychological Measurement, 73(1), 5-26. 
Rodríguez, A., Reise, S. P., \& Haviland, M. G (2015): Applying bifactor statistical indices in the evaluation of psychological measures, Journal of Personality Assessment. doi:10.1080/00223891.2015.1089249

Rodríguez, A., Reise, S. P., \& Haviland, M. G. (2016). Evaluating bifactor models: Calculating and interpreting statistical indices. Psychological Methods, 21(2), 137-150. doi:10.1037/met0000045.

Routt, G., \& Anderson, L. (2011). Adolescent violence towards parents. Journal of Aggression, Maltreatment \& Trauma, 20, 119. doi:10.1080/10926771.2011.537595.

Straus, M. A. (1979). Measuring intrafamily conflict and violence: The Conflict Tactics Scales (CTS). Journal of Marriage and the Family, 41, 75-88. doi:10.2307/351733.

Straus, M. A., \& Fauchier, A. (2007). Manual for the dimensions of discipline inventory (DDI). Durham, NH: Family Research Laboratory, University of New Hampshire. 


\section{Apéndice. CUESTIONARIO DE VIOLENCIA FILIO-PARENTAL, VERSIÓN JÓVENES (C-VIFIP-J), ADAPTACIÓN CHILENA}

Indique con qué frecuencia ha llevado a cabo cada una de las siguientes conductas contra su padre o su madre cuando usted tenía entre 12 y 17 años.

$0=$ Nunca $1=$ Raramente $2=$ Algunas veces $3=$ Bastantes veces $4=$ Muy a menudo

\begin{tabular}{|c|c|c|c|c|c|c|c|c|c|c|}
\hline & \multicolumn{5}{|c|}{ PADRE } & \multicolumn{5}{|c|}{ MADRE } \\
\hline 1. Grité a mis padres. & 0 & 1 & 2 & 3 & 4 & 0 & 1 & 2 & 3 & 4 \\
\hline $\begin{array}{l}\text { 2. Llegué a decir cosas a mis padres como "te odio", "ojalá te } \\
\text { mueras". }\end{array}$ & 0 & 1 & 2 & 3 & 4 & 0 & 1 & 2 & 3 & 4 \\
\hline 3. Insulté a mis padres. & 0 & 1 & 2 & 3 & 4 & 0 & 1 & 2 & 3 & 4 \\
\hline $\begin{array}{l}\text { 4. Dije a mis padres que si yo quería algo, tenían la obligación de } \\
\text { dármelo. }\end{array}$ & 0 & 1 & 2 & 3 & 4 & 0 & 1 & 2 & 3 & 4 \\
\hline $\begin{array}{l}\text { 5. Hice comentarios ofensivos, negativos y/o degradantes a mis } \\
\text { padres. }\end{array}$ & 0 & 1 & 2 & 3 & 4 & 0 & 1 & 2 & 3 & 4 \\
\hline $\begin{array}{l}\text { 6. Cuando veíamos la televisión en casa, exigí a mis padres } \\
\text { controlar el control remoto. }\end{array}$ & 0 & 1 & 2 & 3 & 4 & 0 & 1 & 2 & 3 & 4 \\
\hline 7. Lancé cosas a mis padres. & 0 & 1 & 2 & 3 & 4 & 0 & 1 & 2 & 3 & 4 \\
\hline 8. Golpeé a mis padres con algo que pudiera hacerles daño. & 0 & 1 & 2 & 3 & 4 & 0 & 1 & 2 & 3 & 4 \\
\hline 9. Escupí a mis padres. & 0 & 1 & 2 & 3 & 4 & 0 & 1 & 2 & 3 & 4 \\
\hline 10. Tomé cosas de mis padres sin su permiso. & 0 & 1 & 2 & 3 & 4 & 0 & 1 & 2 & 3 & 4 \\
\hline 11. Di una patada, cachetada y/o puñetazo a mis padres. & 0 & 1 & 2 & 3 & 4 & 0 & 1 & 2 & 3 & 4 \\
\hline 12. Robé dinero a mis padres. & 0 & 1 & 2 & 3 & 4 & 0 & 1 & 2 & 3 & 4 \\
\hline 13. Empujé a mis padres. & 0 & 1 & 2 & 3 & 4 & 0 & 1 & 2 & 3 & 4 \\
\hline $\begin{array}{l}\text { 14. Exigí a mis padres que dejaran lo que estaban haciendo para } \\
\text { que me atendieran. }\end{array}$ & 0 & 1 & 2 & 3 & 4 & 0 & 1 & 2 & 3 & 4 \\
\hline
\end{tabular}

Si ha mostrado alguna de las conductas anteriores, por favor, señale la frecuencia de los motivos por los que actuó de ese modo.

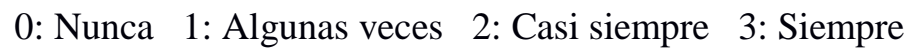

\begin{tabular}{|c|c|c|c|c|}
\hline 1.Por querer llegar más tarde a casa cuando salías por la noche & 0 & 1 & 2 & 3 \\
\hline 2.Para que tu padre/madre te diera más dinero & 0 & 1 & 2 & 3 \\
\hline 3.Para que tu padre/madre te comprara algo que querías & 0 & 1 & 2 & 3 \\
\hline 4.Evitar hacer alguna tarea (recoger tu habitación, ayudar en las tareas de casa...) & 0 & 1 & 2 & 3 \\
\hline 5.Evitar ir a clase, estudiar & 0 & 1 & 2 & 3 \\
\hline 6.Por tu propio carácter & 0 & 1 & 2 & 3 \\
\hline 7.En respuesta a una agresión física (cachetada, puñetazo, empujón....) de tu padre/madre & 0 & 1 & 2 & 3 \\
\hline 8.En respuesta a una agresión verbal (por ejemplo insulto) de tu padre/madre & 0 & 1 & 2 & 3 \\
\hline
\end{tabular}

